\title{
Active Hearing Mechanisms Inspire Adaptive Amplification in an Acoustic Sensor System
}

\author{
José Guerreiro, Student Member, IEEE, Andrew Reid, Joseph C. Jackson, and James F.C. Windmill, \\ Senior Member, IEEE
}

\begin{abstract}
Over many millions of years of evolution, nature has developed some of the most adaptable sensors and sensory systems possible, capable of sensing, conditioning and processing signals in a very power- and size-effective manner. By looking into biological sensors and systems as a source of inspiration, this paper presents the study of a bio-inspired concept of signal processing at the sensor level. By exploiting a feedback control mechanism between a front-end acoustic receiver and back-end neuronal based computation, a nonlinear amplification with hysteretic behavior is created. Moreover, the transient response of the front-end acoustic receiver can also be controlled and enhanced. A theoretical model is proposed and the concept is prototyped experimentally through an embedded system setup that can provide dynamic adaptations of a sensory system comprising a MEMS microphone placed in a closed-loop feedback system. It faithfully mimics the mosquito's active hearing response as a function of the input sound intensity. This is an adaptive acoustic sensor system concept that can be exploit by sensor and system designers within acoustics and ultrasonic engineering fields.
\end{abstract}

Index Terms - bio-inspired acoustics; adaptive sensor system; active hearing; nonlinear amplification; compressive gain; feedback computation; real-time embedded signal processing; prototyping.

\section{INTRODUCTION}

A UDITORY receptors have evolved to be adaptable using feedback mechanisms between mechanical and electrical systems that greatly empower the ability to perceive sounds. Nature has the capacity to exploit and gain awareness of the surrounding environment through the sense of hearing. For instance, some animals use their hearing abilities as a fundamental resource for communication such as humans; bats use it as part of a sophisticated hunting system to locate prey such as moths which counteract against that with adaptive ears that can predict the bats echolocation calls [1]; among other examples [2]. Both vertebrates and invertebrates have followed different evolutionarily pathways, however they share some similarities in respect to the active phenomena of hearing [3]. Generally, amplification and sharp frequency selectivity are considered to be the most important functions taking place at the initial stages of signal conditioning performed by an auditory system. The ability to amplify and tune at specific

This research is funded by the European Research Council under the European Union's Seventh Framework Programme (FP/2007-2013) / ERC Grant Agreement n. [615030].

José Guerreiro, Andrew Reid, Joseph C. Jackson and James F.C. Windmill are members of Centre for Ultrasonic Engineering, Electronic and Electrical frequencies can benefit such a sensory system to separate desired but sometimes weak signals from undesired background noises. Studies based on the vertebrate inner ear report that a healthy hearing system can provide gains up to 40-60 dB [4]. That is thought to be a consequence of energy injection provided by electromotile cells, so called mechanoreceptors, which putatively increase the magnitude of their mechanical inputs in a sort of positive-feedback mechanism. Moreover, the hearing system is more likely to amplify low level sounds and can be less responsive, proportionally, as they become louder. This function is generally known as the compressive nonlinearity that can be achieved by a hearing organ, and is a fundamental mechanism that greatly enhances overall dynamic range. For instance, in humans the dynamic range can be up to $120 \mathrm{~dB}$ when preserved in a healthy condition [5]. Hearing research has also been conducted using insects as an animal model, again providing evidence that their acoustic sensors, neuronal circuits and systems have evolved many interesting properties in terms of power-efficiency, robustness to noise and size-adapted sensor and signal processing mechanisms that best suit their needs [6-7]. Recently, engineers have looked into some insects' ears for inspiration, and a new design trend of acoustic sensors and systems has emerged, exploiting novel directional [8-10] and adaptive sensing capabilities [11].

By following a similar premise, this paper aims to present an adaptive concept of signal processing applied to acoustics performed at the sensor level; an unconventional method to amplify sound. Signals can be amplified in a nonlinear fashion by exploiting a positive-feedback control technique between the front-end acoustic receiver and a back-end computational system, and produce a hysteretic acoustic response result as similarly seen in the mosquito's hearing system thought to be parametric amplification [12].

The assumption is that the active hearing responsiveness to sound is greatly enhanced by synchronized neuronal cells pumping additional energy entrained with a front-end acoustic receiver (antenna) through a positive-feedback system [13-14]. It seems to be an unconventional but advantageous technique to adapt the sensory responsiveness to a desired input stimuli. Therefore, tuning, nonlinear compressive gain, and a hysteretic response of such a sensory system can result [13-14].

Engineering Department, University of Strathclyde, Glasgow, Scotland, UK (emails:\{jose.guerreiro, andrew.reid,joseph.jackson,james.windmill\} @ strath.ac. uk). 
Concurrently, other studies using engineered resonant sensors, circuits and systems, likewise support the use of positivefeedback control techniques, which also enable adaptive nonlinearities in low-powered filtering applications [15], hysteretic behavior applied to speech in noise applications [16], and that the $Q$ factor of sensors can be enhanced exploiting the feedback control technique so-called parametric amplification, for instance, used in atomic force microscopy [17-18]. Therefore, this work aims to exploit the use of an analogous positive-feedback technique at the sensor level - a front-end acoustic receiver that is controlled by a back-end neuronal computational process.

This adaptive sensory system concept may have applications within acoustic and ultrasonic fields, which may require adaptive amplification in a nonlinear fashion. The concept is described using a theoretical model, validated through simulation, and it is prototyped using an experimental setup that enables results in real-time as a proof of concept. This paper is organized as follows: Section II introduces some background knowledge related to the mosquito auditory system that includes sound reception and neuronal processing; Section III describes some background regarding acoustic receivers with emphasis on their physical properties and trade-offs that are set by their design specifications; Section IV and V introduce the concept of an adaptive acoustic sensor system through a theoretical model, respectively; Section VI highlights the simulated and experimental results; and finally Section VII summarizes the main conclusions and outcomes of the study.

\section{THE Mosquito HEARING System}

Mosquitoes exhibit remarkable hearing mechanisms for sound perception. Their ears evolved to be very sensitive sensors to detect the particle velocity component of sound, achieving acute neuronal sensitivities at the base of their frontend receivers [19]. These acoustic receivers are composed of antenna-like structures which can detect sound through the motion of air particles that viscously drags the mechanical structures. Those protrude from an auditory receptor organ where thousands of force sensitive cells also called mechanoreceptor cells reside [20]. When stimulated, the mechanoreceptors can convert the mechanical energy into electrical signaling in a form of spike-type neuronal responses commonly called action potentials. Mosquitoes rely on their hearing system to increase their mating successes. Preferentially, males can detect the sound particle displacements generated by a flying female of $3.5 \mathrm{~nm}$ from a distance of $10 \mathrm{~cm}$ away, within the frequency range of 350-450 $\mathrm{Hz}$ [21]. Intuitively, for a male mosquito to detect and pursue a flying target, its sound receptors may need to transit between two modes of operation: (i) a fast transient time response which allows the sensory system to quickly detect a close by target; (ii) a frequency-selective sensing mode which enhances the intelligibility of sounds generated by a selected/targeted source. A dynamic transition between these two modes of operation might not be achieved by a conventional passive acoustic sensory system. Studies based on the mosquito hearing system report that the presence of a positive-feedback mechanism using an ensemble of synchronized neurons pumping additional energy entrained with the acousto-mechanical response of the front-end acoustic receiver (antenna) to dynamically adapt the overall response of the hearing system, according to an input stimulus presented [13-14], as illustrated in Fig. 1.

This method of sensory adaptation can be very attractive from the engineering point of view for two reasons: firstly exploiting the use of feedback processing at the sensor level might be a reliable technique to allow sensory adaptation [11]; secondly, the process of encoding sound in a form of action potentials (e.g. spike-type signals) might be a simple form of computation with low power requirements [22-25]. Nevertheless, to apply a mechanism to encode sound in a form of action potentials, and the use of a positive-feedback control technique to enhance signal conditioning at the sensor level, first require a clear understanding of, and methods to model those processes.

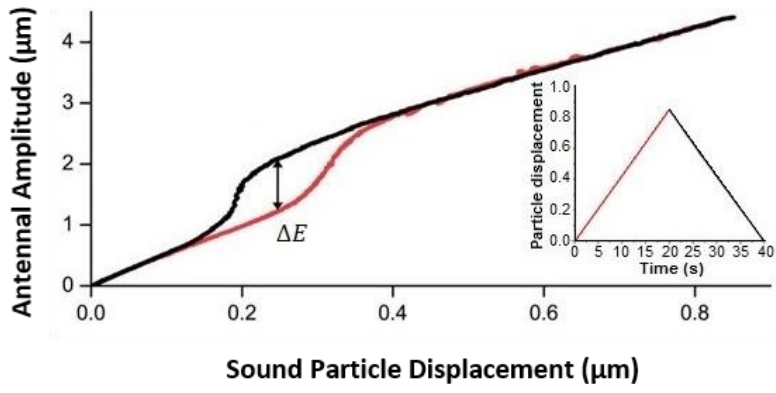

Fig. 1 - Nonlinear response of the mosquito's hearing. Antennal response showing amplification and hysterisis. $\Delta E$ is the rational energy of oscillation between the hysteretically amplified and the initial nonamplified response. (Inset) envelop of an amplitude modulated single-tone sound stimuli used to test the nonlinearities in the antenna's response - increasing (red) and decreasing (black) intensity. Adapted from [13].

\section{A. Neuronal Computation}

Electrical signaling is a fundamental mechanism that underlies many aspects of signal processing and communication between neurons. Biological cells consume energy in order to establish a potential gradient between intracellular and extracellular fluids. Neurons have evolved interesting mechanisms (passive and active) to exploit the electrochemical potential. For instance, neurons can generate electrical signals through ionic influx and efflux of molecules (e.g. sodium $\left(\mathrm{Na}^{+}\right)$, potassium $\left(\mathrm{K}^{+}\right)$, chloride $\left(\mathrm{Cl}^{-}\right)$and calcium $\left.\left(\mathrm{Ca}^{2+}\right)\right)$ across their cell membrane. Different concentrations of ions establish potential gradients between the inside and outside of the cell. Generally, at the equilibrium state (i.e. resting potential) a neuron is able to be stimulated electrically or chemically (i.e. depending on their type and function). Cellular stimulations may alter the ionic gradients across the cell membrane and therefore the cell can hyperpolarize or depolarize. Hyperpolarization occurs when the cell potential becomes more negative than its resting state (negative potential). Depolarization is the opposite response, occurring when the cell increases its potential. These dynamics are normally called the "passive" responses happening at the cellular level. However, when strongly stimulated 
(depolarized), neurons can also become "active" generators of electrical signals, so called action potentials. These are spiketype signals that occur every time the cell potential reaches a certain voltage threshold. After crossing this threshold, a cascade of electrochemical dynamics occurs, resulting in a sudden increase of its potential level, followed by the cellular machinery counteracting that by imposing an automatic control mechanism to discharge (i.e. repolarize) and reset the cell, towards its equilibrium state (resting potential). A neuron can spontaneously generate electrical pulses repetitively as often as input stimuli are presented, however, the cell normally requires a refractory period. That is the time spent by the cell machinery to recover after firing an action potential. Recalibration of the ionic equilibrium is required until the neuron is able to fire again. The action potential is induced by depolarization of the cell, and is sometimes a spontaneous event, in which the duration can vary based upon many factors differing between cell type and function. Typically, firing rates are within the millisecond range. Motivated by these biological mechanisms of signal processing, neuroscientists have developed some mathematical methods which help to study them, leading to the creation of neuronal computational models such as the "Leaky Integrate-and-Fire" (LIF neuron) model [26]. The LIF is a simplified model that resembles the behavior of a single neuron. It ignores the conductances of $\mathrm{Na}^{+}$and $\mathrm{K}^{+}$responsible for the action potential generation which are normally included in more complete neuronal models such as the Hodgkin-Huxley [27]. Instead, the LIF model replaces that by assuming an equivalent membrane's capacitance $(C)$ and resistance $(R)$, and evolving the membrane's voltage potential over time according to the differential equation presented in (1).

$$
C \frac{d V_{m}}{d t}=-\frac{V_{m}}{R}+I, \quad t>0
$$

Solving the equation, by applying the Euler method which considers the approximation: $V_{m}\left(t_{n+1}\right)-V_{m}\left(t_{n}\right) \approx h\left(\frac{d V_{m}}{d t}\right)$, where $h=d t$ (i.e. step size), thus:

$$
V_{m}\left(t_{n+1}\right)=V_{m}\left(t_{n}\right) \cdot\left(1-\frac{d t}{\tau}\right)+R \cdot I\left(t_{n+1}\right) \cdot\left(\frac{d t}{\tau}\right)
$$

$\tau=R$. $C$ represents the membrane's time constant; $V_{m}$ represents the membrane's voltage potential, $I$ is the input stimulus which might be associated to any input current reaching the cell body (i.e. soma). By computing (2) over time $\left(t_{n}\right)$, an action potential can be fired when a voltage threshold is reached. Typically, real neurons hold some time before they are capable to fire again. This refractoriness is normally composed of two periods: a hard (absolute) and a soft (relative) period. A hard refractory period is a time in which a neuron cannot fire absolutely, whereas a soft refractory period is a time during which the threshold is generally more elevated than its steady-state value such that an action potential may not be likely to occur. Overall in a LIF model, an absolute refractory period can generally be set by a constant time whereas a relative refractoriness can be set by a sudden increase (i.e. arbitrarily) of the threshold value, which may decay over time towards its steady-state after each firing. It is shown that the mathematical implementation of the LIF neuron using the Euler method can be very efficient in terms of its computational cost when compared with other methods to compute neuronal models. Refer to [25] for a comparative study of methods to compute neuronal models, including the LIF, Hodgkin-Huxley [27] and Izhikevich [28] models.

\section{B. On-Off Controller}

How can we visualize and perhaps implement this biomechanism using control-systems theory? The simplest and most well-known control mechanism is the On-Off controller. This method of control is based on a continuous comparison between a defined threshold with the input that is presented to the system, which may result in a switching output response. At some degree this might reflect the "all-or-none" behavior of a neuron. However, the response exhibited by a neuron is more sophisticated than purely a switching mechanism. Its dynamics can be self-controlled showing a sort of oscillatory behavior as a consequence of: (i) a growth of some quantity; (ii) until reaching a threshold; (iii) followed by a self-reset. The process can repeat itself in a form of a continuous sequence of cycles being produced by the system. These kind of responses are commonly exhibited by relaxation oscillators that describe many phenomena across different disciplines [29].

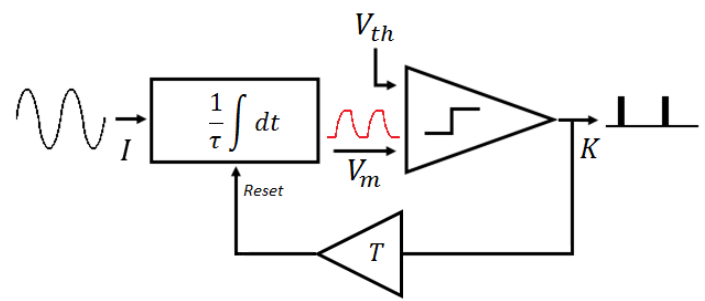

Fig. 2 - Simplified diagram overview of the LIF system, where $\tau$ represents the integrator constant, $T$ is the refractory time (delay) that is used to reset the leaky integrator. $V_{m}$ is the membrane's voltage potential. $V_{t h}$ is the comparator threshold which for the purposes of this study it only assumes a positive value. The output signal of the comparator is composed of square pulses with amplitude $K$ and positive polarity, which can also be referred as the feedback signal to be injected to the front-end acoustic receiver as described in the following sections of this paper. Adapted from [12].

A diagram overview of a LIF system is faithfully represented by Fig. 2. The system is composed of a leaky integrator (linear or not), a comparator (with static or variable threshold) and a reset time delay (constant or not) in the feedback pathway. For instance, by tuning the system for a defined function, pulses can be generated according to the phase of the input signal that is presented to the system. Therefore, the LIF system can then be considered as a smart generator of pulses and perhaps be exploited as a control mechanism for an adaptable sensory system as further described in this paper. A system generator of pulses can also be expressed in Laplace form as described in (3), where $s$ represents the Laplace term $(s=i \omega)$.

$$
L I F(s)=\frac{K \cdot e^{-(\tau . d t) \cdot s}}{\tau_{r . s+1}}-\frac{K \cdot e^{-(W+\tau \cdot d t) \cdot s}}{\tau_{r \cdot s+1}}
$$

$K$ represents the amplitude of the pulse, $\tau . d t$ is the time delay 
before a pulse be generated (e.g. time spent by the integrator function), $\tau_{r}$ is the constant time associated to the rising of the output signal (i.e. pulses generated by a non-ideal driver circuitry) which should be much smaller than $W\left(\tau_{r} \ll W\right)$ in order to provide a reliable square-shaped pulse, and $W$ is the pulse width (duration).

\section{A PASSIVE ACOUSTIC RECEIVER}

Conventionally, the resonant response exhibited by a frontend acoustic receiver resembles the one given by a driven damped harmonic oscillator [30], which for the purposes of this work can simply be expressed by the transfer function in (4).

$$
H(s)=\frac{s \frac{\omega_{0}}{Q}}{s^{2}+s \frac{\omega_{0}}{Q}+\omega_{0}^{2}}
$$

This kind of signal detector can be characterized either by structural mechanical properties such as stiffness $(k)$, mass $(m)$ and dissipation, or by physical properties such as resonance frequency $\left(\omega_{0}=\sqrt{k / m}=2 \pi f_{0}\right)$ and quality factor $(Q$ $\left.=\omega_{0} / \gamma\right)$, where $\gamma$ represents the damping coefficient. The $Q$ factor expresses how quickly the energy supplied to the sensor can be dissipated in it. It means that an underdamped system (high- $Q$ sensor) exhibits a slower temporal resolution compared to an overdamped one (low- $Q$ sensor) that may achieve a faster temporal responsiveness when subject to an applied acoustic stimuli. Additionally, a passive sensor of this kind exhibits linear sensitivity and its bandwidth can be expressed by the ratio between resonance frequency and quality factor, $\Delta \omega=$ $\omega_{0} / Q$. Therefore, that sets a design trade-off between time versus frequency responsiveness of an acoustic sensor, as summarized in Table I.

Table I - Summary of time vs frequency resolution/response of a resonant acoustic sensor.

\begin{tabular}{ccc}
\hline & $\begin{array}{c}\text { Temporal } \\
\text { resolution/response }\end{array}$ & $\begin{array}{c}\text { Frequency } \\
\text { resolution/response }\end{array}$ \\
\hline low- $Q$ sensor & high/fast & low/wide \\
high- $Q$ sensor & low/slow & high/narrow \\
\hline
\end{tabular}

\section{BIO-INSPIRED ADAPTIVE NONLINEAR AMPLIFICATION}

A bio-inspired concept for sensory signal conditioning that exploits feedback computation at the sensor level is proposed and described as follows. It faithfully describes an active process that is inspired by the possible physical basis for the mosquito hearing. From the engineering point of view, this concept can be illustrated by the closed-loop diagram presented in Fig. 3 and its transfer function is expressed by (5).

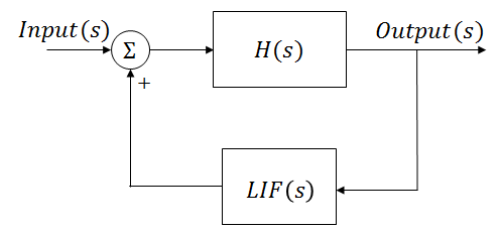

Fig. 3 - Diagram overview of the closed-loop feedback system used to model the concept of active nonlinear amplification. $H(s)$ represents the transfer function of a front-end acoustic receiver (e.g. microphone) and the $L I F(s)$ is the transfer function of a pulse generator (e.g. microcontroller) that is placed in a positive-feedback fashion. Input(s) represent mechanical vibrations due to acoustic energy coupled with the sound receiver structures (e.g. microphone diaphragm) and Output( $s$ ) is the signal readout from the acoustic sensor (e.g. signal resulted from the transduction method used, for instance optical readout using laser can be used to measure the diaphragm displacements).

$$
\frac{\operatorname{Output}(s)}{\operatorname{Input}(s)}=\frac{H(s)}{1-L I F(s) \cdot H(s)}
$$

The concept is based on a feedback system considering two fundamental elements: a front-end acoustic receiver which has the role to detect sound, performing the first stage of signal detection and conditioning (mechanical filtering and transduction of energy from mechanical to an electrical form); and a back-end computational system, which assists in the process to further enhance the sensor responsiveness to a targeted stimulus. The overall response of the sensory system is greatly dependent on the coupling between these two elements (front-end acoustic receiver + back-end computation), which once combined can result in a nonlinear amplification with a hysteretic behavior.

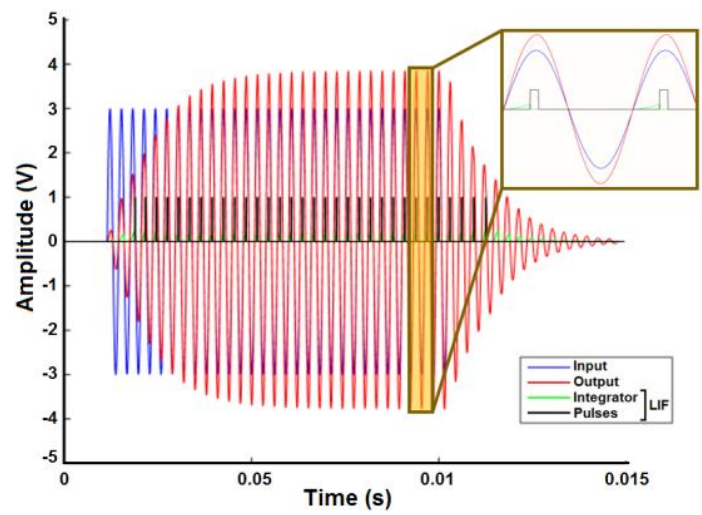

Fig. 4 - Example of the 1:1 resonance entrainment of the input signal (blue graph) with pulses (black graph). Pulses are generated after the integration reaches a defined threshold level. Overall, the coupling of both signals result in an amplified and mechanically filtered output response (red graph) performed at the transducer level.

Theoretically, if the damping of the front-end receiver can be changed dynamically, that might have a consequent effect on its sensitivity to sound (i.e. $Q$ is altered). This principle can be performed through the entrainment of pulsatile energy, a form of squared pulses that are injected to the front-end acoustic receiver in a synchronized and cycle-by-cycle manner exploiting 1:1 resonance as illustrated in Fig. 4. It means that pulses are entrained with the input signal at the same frequency. Therefore, an amplified and filtered response can likely result from an in-phase summation of signals that are mechanically coupled by the front-end acoustic sensor. For instance in a real system, it means that the mechanical vibrations induced by the input sound waves into a microphone's diaphragm are likely added to the vibrations generated by the pulsatile actuation imposed by a feedback system mechanism (e.g. microcontroller). Therefore, the summation of signals is done at the mechanical level of the microphone itself (refer to our 
experimental setup in Section VI B for a practical implementation of this concept). Additionally, under certain conditions it may behave like a critical acoustic sensor - an active system that operates near the oscillatory instability [29]. This behavior has been reported from within several studies on biological sensors, and has also been included within state-ofthe-art auditory models [31].

\section{THEORETICAL MODEL}

This section summarizes the theoretical model of the purposed sensory system that is simulated through a numerical approach using MatLab R2014b. In order to better understand the system's dynamics, a model using a front-end acoustic receiver described by (4) is placed within a positive-feedback loop controlled by a computational function given by (2) and exploiting the On-Off mechanism presented in Fig. 2. This modelling approach is directly derived from the mathematical implementation of (2) and (4) computed in a recursive way as a closed-loop system. It is important to note that, (4) is mapped from the analog-to-digital domain using bilinear transformation and implemented as an IIR filter using a biquadratic topology. The concept is tested using noise-free synthetic signals with $d t=1 \mu s$ that is the time-step resolution. The resonant frequency of the system is $f_{0}=3.3 \mathrm{kHz}$ and the LIF model features include: $T=0.303 \mathrm{~ms}, \tau=10 \mathrm{~ms}$ and $W=20 \mu \mathrm{s}$.

\section{A. Stability}

An evident consequence of using a control mechanism imposing pulses in a positive-feedback with a resonant sensor is how the stability of this system can vary under certain conditions. A system is found stable if its output tends to converge to an equilibrium state $(\operatorname{LIF}(s) . H(s)<1)$. It becomes unstable if the output appears to diverge without bound $(\operatorname{LIF}(s) . H(s)=1)$. A system can also be classified as critically stable when the output converges to a continuous and endless oscillatory state $(\operatorname{LIF}(s) . H(s)>1)$. Testing the system's stability is then a preliminary task in order to understand the nature of its behavior under certain conditions.

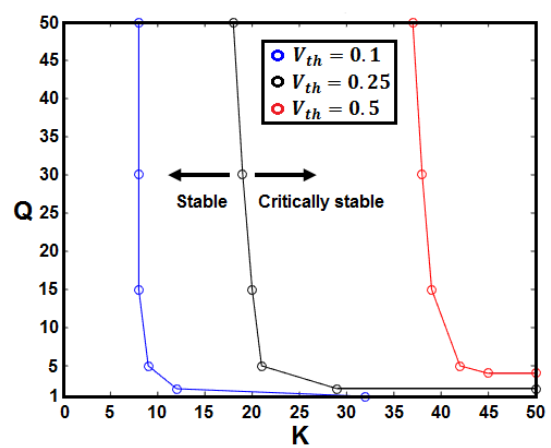

Fig. 5 - Stability diagram of the sensor system obtained by numerical simulation (using an impulse response analysis). $Q$ represents the quality factor of the front-end acoustic receiver and $K$ is the feedback signal gain.

Fig. 5 shows the stability diagram calculated from numerical implementation of the closed-loop system presented in Fig. 3 while varying $Q, K$ and $V_{t h}$. As previously described, the $Q$ factor expresses how quickly the energy can be dissipated by the front-end acoustic receiver, meaning that a sensor with a high- $Q$ may start to oscillate by itself when a high feedback signal gain $(K)$ configuration is applied to it, whereas a low- $Q$ sensor may show a higher level of convergence under the same $K$ conditions. Additionally, $V_{t h}$ represents the threshold that sets the feedback operation, which can also play a key role in the system's stability, since it can determine the position where the stability curve is located (Fig. 5).

In order to have a better characterization of a given system's operating point, Fig. 6 shows the bifurcation diagram obtained from a test while varying $K$, which sets the amplitude of the pulses, assuming a front-end acoustic receiver with $Q=30$ (i.e. for our custom-built MEMS microphone [32] used in the experimental setup as presented in later sections) and feedback threshold, $V_{t h}=0.25 \mathrm{~V}$. The simulated system presents a Hopf bifurcation around the $\beta=19$ point. Briefly, the Hopf bifurcation is defined as the critical region or point $(\beta)$ where the system transits from a stable to an oscillating unstable or critically stable operating regime (refer to [4] for further details about the Hopf bifurcation related to hearing research). To ensure a stable system operation, it should function under the left side of the $\beta$ point, and therefore the $K$ chosen should obey that condition for a defined $V_{t h}$.

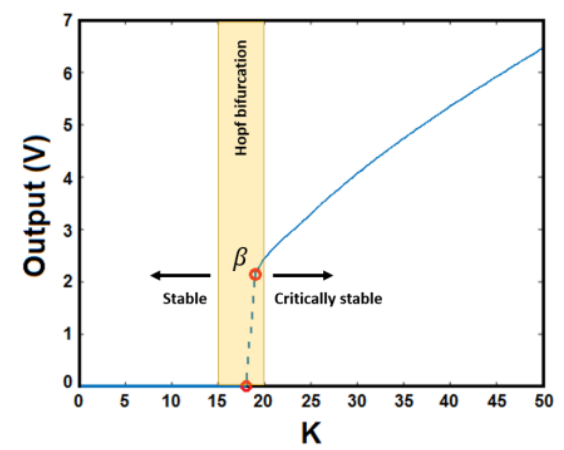

Fig. 6 - Bifurcation diagram of the sensor system obtained by numerical simulation using an impulse response analysis within the following conditions: $Q=30, V_{t h}=0.25 \mathrm{~V}$ and varying $K$.

\section{B. Nonlinear Compressive Gain}

One of the advantages of the feedback technique exploited in this study is the fact that it can provide a nonlinear compressive gain to the overall sensor response. For instance, when $K$ value is higher than the input signal itself, the contribution of the energy added to the system dynamics, from the pulsatile feedback signal, is higher than the reverse situation - the input amplitude is higher than the feedback energy injected, therefore the system can exhibit a nonlinear compressive gain as illustrated in Fig. 7.

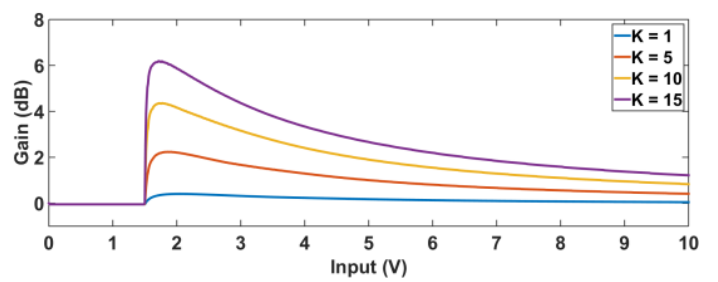

Fig. 7 - Example of the nonlinear compressive gain that can be provided from the sensor system using the following conditions: input signal frequency $f_{\text {in }}=f_{0}=3.3 \mathrm{kHz}, Q=30, V_{\text {th }}=0.25 \mathrm{~V}$ for $K=1,5,10$ and 15 .

The system is operated within its stable regime using a 1:1 
resonance mode (refer to Fig. 4), hence the feedback signal gain should obey $K<19$, when $Q=30$ and $V_{t h}=0.25 \mathrm{~V}$. Assuming $K=15$, then a stable operation of the system is ensured that results in a peak-gain given by the system of about $6.2 \mathrm{~dB}$ at $1.76 \mathrm{~V}$ of the input amplitude (refer to Fig. 7). Therefore, the feedback contribution to the overall gain (Vout/Vin) is seen as nonlinear and it is also dependent on the input signal amplitude following an experimentally determined exponential relationship expressed by (6), where $x$ represents the input signal amplitude $(\mathrm{V}), \delta$ is the peak-gain $(\mathrm{dB}), \theta$ is defined as the input amplitude for an achieved peak-gain $(\delta)$, and $\alpha=1$ (maxGain/minGain) gives the gain compression rate factor.

$$
\operatorname{Gain}_{[d B]}=\left\{\begin{array}{cl}
\delta \times e^{-\alpha(x-\theta)} & , x \geq \theta \\
0 & , x<\theta
\end{array}\right.
$$

This is a simplified equation used as a fitting curve to express the gain after a threshold, which follows an exponential decay when the input amplitude is increased, and vice-versa. It is important to note that Vout is equal to Vin when the system is without feedback operation as such Gain $=0 \mathrm{~dB}$. Table II summarizes some of the gain features using different feedback thresholds. It can be seen that the system while operated under the stable region can achieve a peak-gain of $7.03 \mathrm{~dB}$ when $K=$ 35 and $V_{t h}=0.5$. However, the maximum compression rate factor is $\alpha=0.31$ obtained when the feedback signal gain and threshold are reduced to $K=5$ and $V_{t h}=0.1$, respectively. It highlights that the feedback contribution to the overall gain has a bigger impact at low levels of the input stimuli, as the compression of the gain response arises. $V_{t h}$ can also influence the overall gain given by the system, since it affects the timing of the pulses entrained with a given input signal. For instance, when $V_{t h}=0.5$, the system is more likely to fire pulses closer to the end of the input cycle, since it takes more time for integration to reach the threshold level, than for $V_{t h}=0.1$, which is more likely to set the firing at the beginning of the input cycle, for the same given $K$. However, a perfect locking appears when the pulses are entrained at the middle of the input cycle, thus the contribution to the overall gain can be maximized. It means that each configuration $\left(V_{t h}\right.$ and $K$ ) has its own "best" input amplitude for which it maximizes the system's response.

Table II - Summary of the gain factors obtain under different feedback operations.

\begin{tabular}{ccccc}
\hline$V_{t h}(\mathrm{~V})$ & $K(\mathrm{~V})$ & $\theta(\mathrm{V})$ & $\alpha$ & $\delta(\mathrm{dB})$ \\
\hline 0.1 & 5 & 0.69 & 0.31 & 5.28 \\
0.25 & 15 & 1.76 & 0.20 & 6.20 \\
0.5 & 35 & 3.41 & 0.13 & 7.03 \\
\hline
\end{tabular}

\section{Rise time}

Another important property of the positive-feedback technique exploited in this study is related to the fact that it can alter the effective time response of the overall system when subject to a step input stimulus. The rise time without feedback operation is $18.2 \mathrm{~ms}$, measured at $98 \%$ of the peak amplitude for an acoustic receiver with $Q=30$. Table III presents a summary of the rise times of the system when operated under the influence of feedback computation, where $\Delta$ represents the minimum rise time that can be achieved by the system (milliseconds) and $\sigma$ is the input amplitude (V) that is maximized in terms of the rise time under the defined system configuration set by $V_{t h}$ and $K$. Therefore, if a threshold detection method is required by a given application, the use of the positive-feedback process can provide a faster responsiveness $(\sim 4.5 \mathrm{x})$ under these operating conditions.

\begin{tabular}{cccc}
\multicolumn{4}{c}{ Table III -Summary of the rise time for different feedback operations. } \\
\hline$V_{t h}(\mathrm{~V})$ & $K(\mathrm{~V})$ & $\sigma(\mathrm{V})$ & $\Delta(\mathrm{ms})$ \\
\hline 0.1 & 5 & 1.6 & 4.2 \\
0.25 & 15 & 3.9 & 3.9 \\
0.5 & 35 & 8.2 & 3.6 \\
\hline
\end{tabular}

\section{Hysteretic response}

The input-output relationship of the studied sensory system follows a distinct nonlinear response, dependent on whether the input amplitude is increasing or decreasing. A system showing this kind of behavior is said to have a hysteretic output response. Hysteresis commonly originates due to the on-off feedback control mechanism applied to a system. It can be seen that the feedback operation imposes a switching behavior to the overall system response, which is mainly dependent on the threshold value $V_{t h}$, used. Therefore, a hysteretic behavior is likely to appear as a direct consequence of the control mechanism exploited in this work. The control process is a threshold based computation that can lead to a bistable behavior of the overall sensory system. Sensors that show hysteresis are nonlinear systems. For the purposes of this study it is assumed that hysteresis is a consequent feature of the purposed concept, which may or may not be exploited for a given application, and it is not seen as a drawback of the concept. Biological sensors also show hysteresis, which is exploited as a useful feature, such that it can be seen as a synonym of intrinsic residual memory of the sensory system to a previous targeted signal and perhaps can provide immunity to noise [1].

\section{EXPERIMENTAL RESULTS}

This section presents the system's output response obtained from the implementation of the theoretical model, as used to characterize and test the behavior of the sensory system while applying standard signals. It can be used to further validate the response of the concept obtained from a physical prototyped implementation of this system obtained experimentally.

\section{A. Overall response of the system using numerical simulation}

Fig. 8 shows the dynamic adaptations of the overall sensor system response under the following test conditions: $f_{\text {in }}=f_{0}=$ $3.3 \mathrm{kHz}, Q=30, V_{t h}=0.25 \mathrm{~V}, T=0.303 \mathrm{~ms}, \tau=d t=20 \mu \mathrm{s}, K$ $=10$ and $W=20 \mu s$. That provides a gain of about 1.52 (gain = $3.63 \mathrm{~dB})$ under active feedback computation $\left(\mathrm{A}_{2} \& \mathrm{~B}_{2}\right)$. Fig. 9 shows the hysteretic response of the overall sensory system when the input stimulus follows a different amplitude tendency (increased and decreased). 

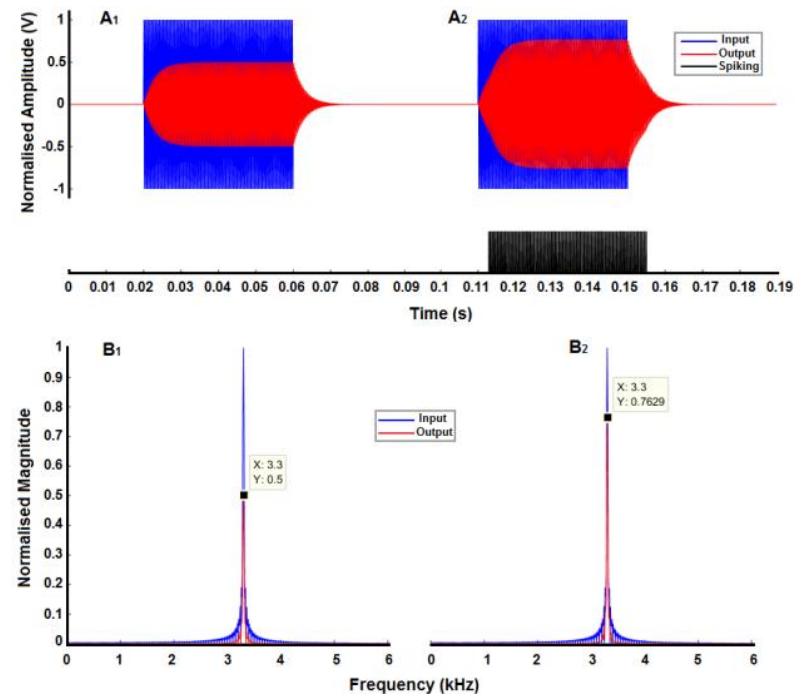

Fig. 8 - Example of an amplified response of the sensory system obtained by simulation. Time and frequency response of a single-tone input (blue); output response of the sensory system (red) without- $\left(\mathrm{A}_{1} \& \mathrm{~B}_{1}\right)$ and with $\left(A_{2} \& B_{2}\right)$ phase-locked pulses (black). The black signal trace in $A_{2}$ is rescaled for the sake of clarity.
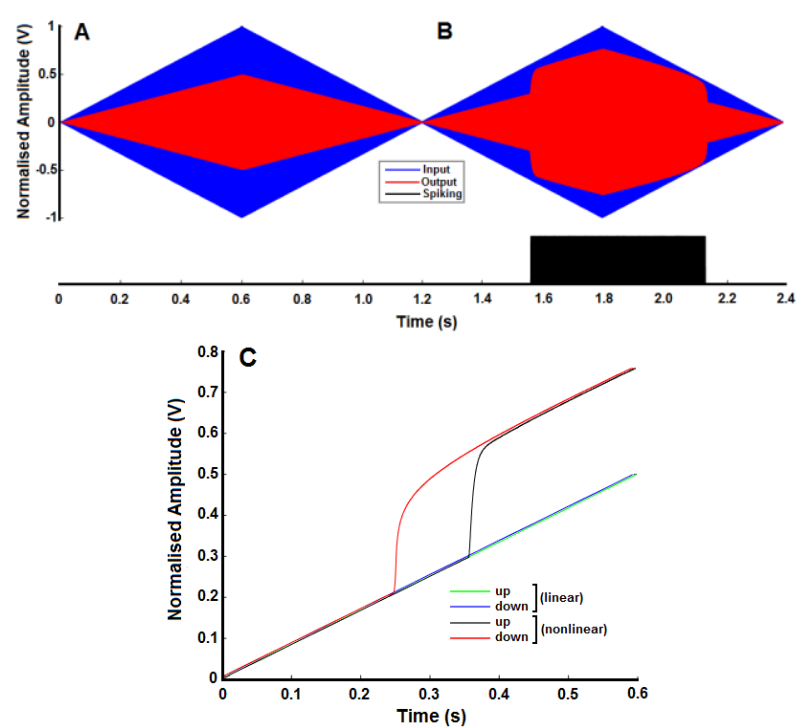

Fig. 9 - Example of the hysteretic response of the sensory system obtained by simulation using an amplitude modulated input signal, (A) without and (B) with feedback control; (C) linear vs nonlinear response of the sensor system. The black signal trace in B is rescaled for the sake of clarity.

\section{B. Response of the sensor system obtain from the experimental setup}

The concept is also prototyped using a custom-built experimental setup presented in Fig. 10, which can perform real-time computation and provide a proof of concept of this parametric amplification applied to a MEMS microphone. That is meant to be a direct implementation of the theoretical model presented in Section IV and V in a practical manner. The system is prototyped using an electromechanical setup as following described: firstly, the function $(H(s))$ of a front-end acoustic receiver is performed by a MEMS microphone. The design and characterization of this device can be accessed in [32], where results of finite element modeling and practical experimentation are reported. Some of the device's main features include: its resonance frequency around $3.3 \mathrm{kHz}$ and the $Q$ factor of 30 ; secondly, the element of feedback computation and control $(L I F(s))$ is implemented through an embedded system setup. By computing a software routine of the method described previously by (2), the embedded system (e.g. microcontroller) is able to generate pulses to be in-phase with a targeted input stimuli reaching the microphone's diaphragm. Feedback signals (e.g. pulses) are driven to the capacitive port of the MEMS microphone.

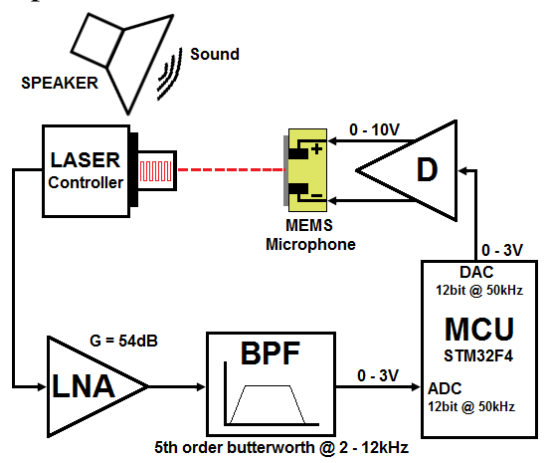

Fig. 10 - Schematic of the custom-built embedded system used to enable the experimental setup. A more complete description of circuits and systems can be found in [11], and refer to [32] for details about the MEMS microphone design used in this experimental setup).

Highlights of the embedded system setup features include: an optical readout from the microphone's diaphragm displacements, signals are additionally conditioned using a custom-built analog circuit, and acquired and processed using a digital computational unit based on the STM32F4 microcontroller (refer to [11] for further details about the experimental setup). The purpose-built sensor system is then tested by experimentation using the parameters that are configured as follows: input acoustic signals with frequency $f_{\text {in }}=f_{0}=3.3 \mathrm{kHz}$ are played by a speaker; and $V_{t h}=0.25 \mathrm{~V}, T$ $=0.303 \mathrm{~ms}, \tau=10 \mathrm{~ms}, d t=20 \mu \mathrm{s}, K<7 \mathrm{~V}$ and $W=20 \mu \mathrm{s}$.
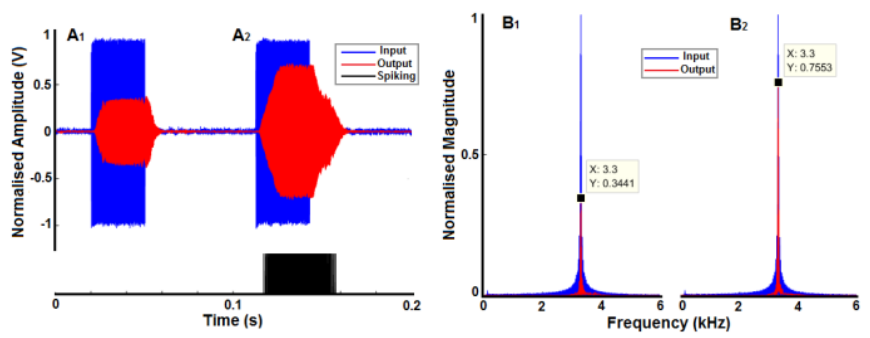

Fig. 11 - Adaptive response of the purpose-built sensor system setup. Time and frequency response of the system for a single-tone acoustic input at 3.3 $\mathrm{kHz}$ (blue); output response of the sensor system (red) without- $\left(\mathrm{A}_{1}, \mathrm{~B}_{1}\right)$ and with- $\left(\mathrm{A}_{2}, \mathrm{~B}_{2}\right)$ feedback contribution (black) with $K=5 \mathrm{~V} @ V_{t h}=0.25 \mathrm{~V}$. The black signal trace is rescaled for the sake of clarity. Adapted from [12].

Fig. 11 presents the response of the sensory system setup showing an output amplification $\left(\mathrm{A}_{2}\right.$ and $\left.\mathrm{B}_{2}\right)$ of about 2.2 greater (gain $=6.85 \mathrm{~dB}$ ), and the system's responsiveness to a step input stimuli is also enhanced (with rise time measured: $\Delta$ $=5.5 \mathrm{~ms})$, when compared with its passive response $\left(\mathrm{A}_{1}\right.$ and $\mathrm{B}_{1}$ - with rise time measured: $\Delta=16.7 \mathrm{~ms}$ ); Fig. 12 shows the response of the system to an amplitude modulated input signal with sound level being increased and decreased consecutively; 
with- (B) and without- (A) feedback control operation, respectively. When the pulses are in-phase with the input signal, the output response shows amplification as predicted by the theoretical model and simulation, and also exhibits similar behavior to that reported for the mosquito's hearing system response [13]. Refer to Fig. 9 for comparisons with the results from the simulation, and Fig. 1 for visual comparisons with the mosquito's hearing response.
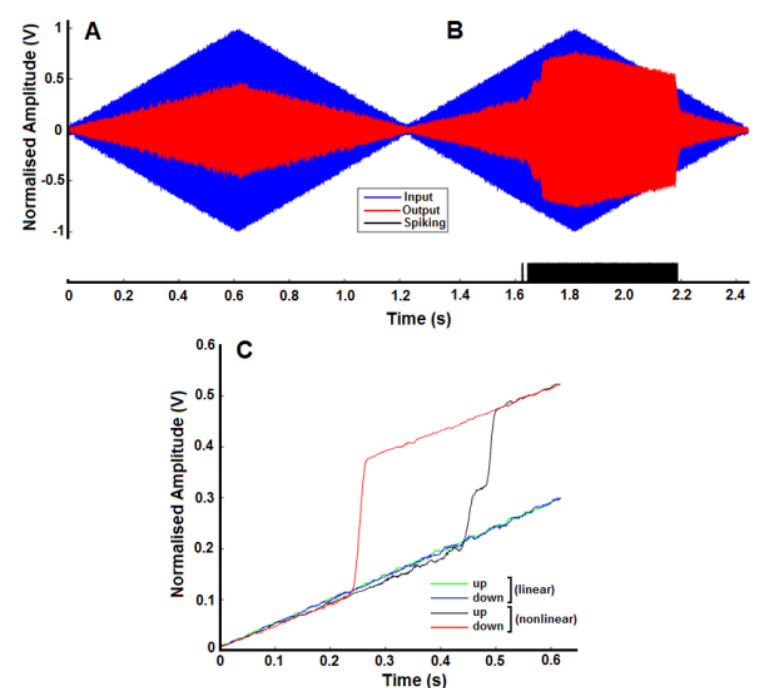

Fig. 12 - Hysteretic response of the purpose-built sensor system setup. The sound level at $3.3 \mathrm{kHz}$ (blue) is increased and decreased consecutively: (A) showing linear response - without feedback; and (B) showing hysteretic nonlinear response with feedback $K=3 \mathrm{~V} @ V_{t h}=0.25 \mathrm{~V}$; (C) linear vs nonlinear response of the sensory system showing the amplification and the hysteretic behavior of the system. The black signal trace in B is rescaled for the sake of clarity. Adapted from [12].

During the experimental tests, the system's operating regime is located within the stable zone. However, it is important to note that the stable region of the overall setup is found after experimentation by tuning the feedback signal gain. Experimentally, the critical region/point of the purpose-built system is reached when $K$ is approximately $7 \mathrm{~V}$ at $V_{t h}=0.25 \mathrm{~V}$. Therefore, in practical terms the critical region/point is reached at smaller $K$ amplitudes when compared to what is predicted by simulation $\left(K=19\right.$ at $\left.V_{t h}=0.25\right)$. In practice, the critical point is expected to be shifted since the positive-feedback system used is greatly affected by the background noise surrounding the experimental setup. It means that, noise can affect the system dynamics in several ways. For instance, noise within the system may impose some jitter to the feedback operation likely seen at the transitory states, as seen in Fig. $11 \mathrm{~A}_{2}$ - the pulse train (black trace) is kept active for a longer time at the downward slope of the system's response, which is not seen in a noise-free simulation - Fig. $8 \mathrm{~A}_{2}$. Additionally, when the system is operating near its threshold voltage, noise can make the system transition between the on-off states momentarily (Fig. 12 B - black trace). This may result in intermediate output fluctuations as the system jumps between the two states (Fig. $12 \mathrm{C}$, black trace onset). There is evidence that this is a separate state, which appears to be at the junction of stability between the two states.

\section{CONCLUSIONS}

The sensitivity of a passive acoustic sensor is greatest when operated at the resonance frequency, and that is also proportionally dependent on the $Q$ factor. Physical constraints are imposed by the sensor's design on the $Q$ factor that can be achieved, such that one might not have much flexibility and control over these parameters when and after designing an acoustic sensor. Therefore, this paper presents a method to provide adaptive nonlinear amplification capabilities at the sensor level. The concept is theoretically described through a model and it is validated experimentally through simulation and physically prototyped using a custom-built setup as a proof of concept. It is clear that the positive-feedback mechanism exploited in this study can enhance signal conditioning at the sensor level, namely amplification and fast sensory responsiveness to sound when the sensor is subject to a step input stimulus. However, in a real scenario this type of system dynamics can be highly influenced by the noise surrounding the setup. Future investigations can address this issue, for instance by adding noise at the simulation level in order to have a better prediction of the system's dynamics in the presence of different types of noise (random noise or any competitive signals that can be generated by electrical or acoustic sources). Moreover, the biological reports [13-14] that inspired this work describe the mosquito hearing response as greatly enhanced due to the "synchrony through twice-frequency forcing" $-2: 1$ resonance mode. It should be noted that this study only explored the use of 1:1 resonance mode (one pulse per cycle with positive polarity), however, one might hypothesize about the response of the proposed sensory system if configured at 2:1 resonance mode (entrainment at twice per cycle - one pulse phase-locked in the positive cycle of the input signal and another pulse phaselocked in its negative cycle with compatible polarity, respectively): (a) it might drag the $\beta$ point towards a lower value of $K$, for the same given threshold value as used with 1:1 mode, meaning that the system might enter in a self-oscillatory condition quicker and at lower feedback signal gain used; (b) the overall performance would benefit from the 2:1 mode in terms of the gain and time response/resolution that can be achieved, since the feedback path can then supply twice the energy per cycle when compared with 1:1 mode. Future investigations can address this modality further, which may add another level of versatility to the system outcomes. Additionally, one may hypothesize that the feedback parameters such as $V_{t h}$ and $K$, which were kept as constant values in this study, can assume variable conditions (e.g. be adaptive), for instance, evolving dynamically to put the system operating point "always" at its best signal-to-noise ratio condition as natural sensors and systems do. This work aims to support the positive cross-disciplinary synergy between biology and engineering based on previous and ongoing bio-inspired research studies. It provides a proof of concept of a bio-inspired acoustic sensor system that can potentially be exploited by both the sensors and the circuits-and-systems communities. The electronics embedded in this prototyped sensor-system are generic and they are mostly based on discrete-level components, therefore, future developments of this work might include bio-inspired electronics based on ultra-low-power IC design techniques [33]. In the near future, one might predict that 
acoustics and ultrasonic engineering are the most likely domains to get this concept through a more advanced and matured stage of implementation, when requirements for adaptation are needed of a sensor, featuring enhanced sensitivity and faster responsiveness to target signals of interest.

\section{REFERENCES}

[1] Windmill, J.F.C., Jackson, J.C., Tuck, E.L. and Robert, D., "Keeping up with bats: Dynamic auditory tuning in a moth". Current Biology, 16(24): 2418-2423, 2006.

[2] Kern, A., Stoop, R., Gopfert, M., Smirnov, D. A., Bezrucko, B. B., "Theory signal processing in insect hearing organs". IEEE Circuit Theory and Design, 2005.

[3] Robert, D. and Hoy, R. R., "Chapter 7: Auditory Systems in Insects". Invertebrate Neurobiology, Cold Springer Harbor Lab Press, 2007.

[4] A. J. Hudspeth. "Making an Effort to Listen: Mechanical Amplification in the Ear". Neuron, 59(4): 530-545, 2008.

[5] K. Dierkes, B. Lindner and F. Julicher. "Enhancement of sensitivity gain and frequency tuning by coupling of active hair bundles". PNAS, 105(48): 18669-18674, 2008.

[6] G. S. Pollack, A. C. Mason, A. N. Popper and R. R. Fay. "Insect Hearing". Springer, 2016.

[7] Robert, D., Mhatre, N. and McDonagh, T., "The small and smart sensors of insect auditory systems". Proceedings of IEEE Sensors 2010.

[8] R.N. Miles and R.R. Hoy. "The Development of a Biologically-Inspired Directional Microphone for Hearing Aids". Audiology Neurotology, 11(2): 86-94, 2006.

[9] Andrew Reid, James F.C. Windmill and Deepak Uttamchandani. "BioInspired Sound Localization Sensor with High Directional Sensitivity". Elsevier Procedia Engineering, 120: 289-293, 2015.

[10] Yansheng Zhang, Ralf Bauer, James F.C. Windmill and Deepak Uttamchandani. "Multi-Band Asymmetric Piezoelectric MEMS Microphone Inspired by the Ormia Ochracea". IEEE 29th International Conference on MEMS, 1114-1117, 2016.

[11] José Guerreiro, Joseph C. Jackson and James F.C. Windmill. "Simple Ears Inspire Frequency Agility in an Engineered Acoustic Sensor System". IEEE Sensors Journal, 17(22): 7298-7305, 2017.

[12] José Guerreiro, Andrew Reid, Joseph C. Jackson and James F.C. Windmill. "Bio-inspired Active Amplification in a MEMS Microphone using Feedback Computation". Proceedings IEEE Biomedical Circuits and Systems Conference 2017

[13] Joseph C. Jackson, James F.C. Windmill, Victoria G. Pook and Daniel Robert, "Synchrony through twice-frequency forcing for sensitive and selective auditory processing". PNAS, 106(25): 10177-10182, 2009.

[14] Windmill, J. F.C., Jackson, J.C., Pook, V.G. and Robert, D., "Frequency doubling by active in vivo motility of mechanosensory neurons in the mosquito ear". R. Soc. Open sci. 5:171082, 2018.

[15] C. D. Salthouse and R. Sarpeshkar, "Jump resonance: A feedback viewpoint and adaptive circuit solution for low-power active analog filters", IEEE Trans. Circuits and Systems, 53(8): 1712-1725, 2006.

[16] K. Aono, et al., "Exploiting jump-resonance hysteresis in silicon auditory front-ends for extracting speaker discriminative formant trajectories", IEEE Trans. Biomedical Circuits and Systems, 7(4): 389-400, 2013.

[17] Rodriguez, T. and Garcia, R., "Theory of Q control in atomic force microscopy", Applied Physics Letters, 82(26): 4821-4823, 2003.

[18] Prakash, G., Hu, S., Raman, A. and Reifenberger, R., "Theoretical basis of parametric-resonance-based atomic force microscopy". Phys. Rev. B 79, 094304, 2009.

[19] M. C. Gopfert and D. Robert. "Nanometre-range acoustic sensitivity in male and female mosquitoes". Proc. R. Soc. Lond. B. 267, 453-457, 2000.

[20] K. S. Boo and A. G. Richards. "Fine structure of the scolopidia in Johnston's organ of female Aedes aegypti compared with that of a male." J. Insect Physiol. 21, 1129-1139, 1975

[21] Joseph. C. Jackson and D. Robert, "Nonlinear auditory mechanism enhances female sounds for male mosquitoes". PNAS, 103(45): 16734$16739,2006$.

[22] Lazar, A.A., "Time encoding with an integrate-and-fire neuron with a refractory period". Neurocomputing, 58(60): 53-58, 2004.

[23] Alvarado, A. S., Rastogi, M., Harris, J. G., Principe, J., "The integrateand-fire sampler: A special type of asynchronous $\Sigma-\Delta$ modulator". IEEE Circuits and Systems (ISCAS) 2011
[24] Varghese, V., Molin, J. L., Brandli, C., Chen, S. and Cummings, R., "Dynamically reconfigurable silicon array of generalized integrate-andfire neurons". IEEE Biomedical Circuits and Systems Conference, 2015.

[25] M. J. Skocik and L. N. Long. "On the Capabilities and Computational Costs of Neuron Models". IEEE Trans. Neural Netw. and Learning Systems, 25(8):1474-1483, 2014.

[26] F. Gabbiani and S. J. Cox, "Chapter 10 - Reduced Single Neuron Models," Mathematics for Neuroscience $1^{\text {st }}$ edition, Elsevier, 2010.

[27] A. L. Hodgkin and A. F. Huxley, "A quantitative description of membrane current and its application to conduction and excitation in nerve." $\mathrm{J}$ Physiol, 117:500-544, 1952.

[28] E. M. Izhikevich. "Simple model of spiking neurons". IEEE Trans. Neural Netw., 14(6): 1569-1572, 2003

[29] Pikovsky A., Rosenblum M., and Kurths J., "Synchronization: A Universal Concept in Nonlinear Sciences", Cambridge Univ. Press, 2001.

[30] Rossing, T. (Ed.) and Chaigne, A., "Chapter: 22 - Structural Acoustics and Vibrations". Handbook of Acoustics, $2^{\text {nd }}$ Edition. Springer, 2014.

[31] M. Rudnicki, O. Schoppe, M. Isik, F. Volk and W. Hemmert, "Modeling auditory coding: from sound to spikes", Springer, Cell Tissue Res (2015) $361: 159-175$

[32] José Guerreiro, Andrew Reid, Joseph C. Jackson and James F.C. Windmill. "Towards the Development of a Frequency Agile MEMS Acoustic Sensor System". Proceedings IEEE Sensors Conference 2017.

[33] Rahul Sarpeshkar. "Ultra-Low Power Bioelectronics: Fundamentals, Biomedical Applications, and Bio-Inspired Systems". Cambridge University Press, 2010.

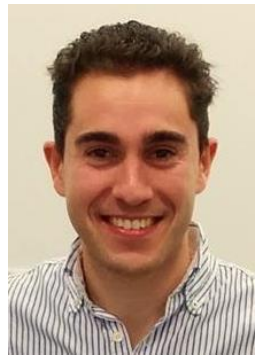

José Guerreiro (S'15) was born in Lisbon, Portugal, in 1988. He received the B.S. and M.S. degrees in Electronic and Telecommunications Engineering from High Institute of Engineering of Lisbon in 2013, and currently is undertaking a Ph.D. degree in Electronic and Electrical Engineering at the University of Strathclyde, Glasgow, United Kingdom.

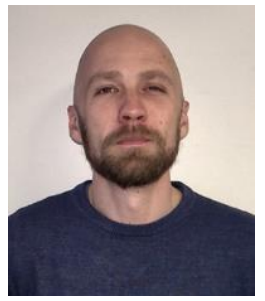

Andrew Reid is a Research Associate in the Department of Electronic and Electrical Engineering at the University of Strathclyde, Glasgow. He has recently completed a $\mathrm{PhD}$ on bio-inspired sound localization and continues to research and develop bio-inspired acoustic sensors. His research focus is on sound-localization techniques and directional hearing.

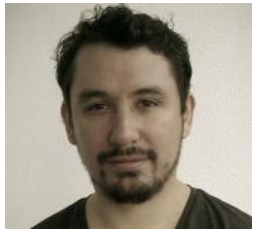

Joseph C. Jackson is a Lecturer in Electronic and Electrical Engineering at University of Strathclyde, based in the Centre for Ultrasonic Engineering. His research interests cover a wide range of subjects, such as the physical basis for hearing, sound production and reception in biology and engineering, and bio-inspired transducer and signal design.

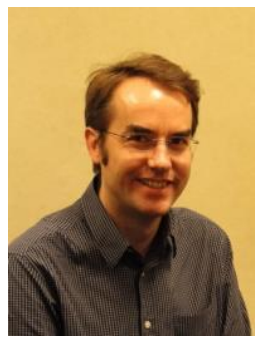

James F. C. Windmill (SM'17) is a Professor in the Department of Electronic and Electrical Engineering at the University of Strathclyde, Glasgow, United Kingdom. He has over 18 years of research and development experience in the areas of sensors and hearing systems. His research interests are in the field of biologicallyinspired acoustic systems, from the fundamental biology to various engineering application topics. 